\author{
Przemysław WIELENTEJCZYK ${ }^{1}$ \\ Roman LEWANDOWSKI ${ }^{2}$
}

\title{
WPLYW WYŻSZYCH HARMONICZNYCH NA NIELINIOWE DRGANIA BELEK WYKONANYCH Z MATERIAŁU KELVINA-VOIGTA
}

\begin{abstract}
W pracy analizuje się geometrycznie nieliniowe drgania ustalone belek wymuszone siłami harmonicznie zmiennymi w czasie. Belki wykonane są z materiału lepkosprężystego opisywanego za pomocą modelu Kelvina - Voigta. Do opisu geometrycznej nieliniowości deformacji zastosowano teorię von Karmana oraz teorię belek Eulera - Bernoulliego. Pominięto wpływ osiowych sił bezwładności oraz założono nieprzesuwność osiową końców belek. Sformułowano związki fizyczne wyrażone za pomocą sił przekrojowych i uogólnionych odkształceń. Równania ruchu oraz równania amplitud zapisano stosując metodę prac wirtualnych, metodę bilansu harmonicznych oraz metodę elementów skończonych. Nieliniowe równania amplitud rozwiązano metodą kontynuacji. W rozwiązaniach okresowych równań ruchu uwzględniono wyższe harmoniczne, co pozwala na analizę rezonansów pobocznych, ale znacząco skomplikowało sformułowanie i rozwiązanie problemu. Za pomocą krzywych rezonansowych przedstawiono dynamiczne zachowanie się belek wykonanych z materiału Kelvina - Voigta. Wyniki porównano z rozwiązaniami dla belek sprężystych. Przeanalizowano istotność wyższych harmonicznych w opisie drgań nieliniowych.
\end{abstract}

Słowa kluczowe: model Kelvina-Voigta, geometryczna nieliniowość belek, MES, drgania ustalone, analiza wyższych harmonicznych

\section{Wprowadzenie}

Celem pracy jest analiza geometrycznie nieliniowych drgań ustalonych belek wymuszonych siłami harmonicznie zmiennymi w czasie $\mathrm{z}$ uwzględnieniem w rozwiązaniu wyższych harmonicznych. Przyjęto do badań belki wykonane z materiału lepko-sprężystego definiowanego jako klasyczny model reologiczny Kelvina-Voigta. Rezultaty zostały przedstawione w postaci krzywych rezonan-

\footnotetext{
${ }^{1}$ Autor do korespondencji / corresponding author: Przemysław Wielentejczyk, Politechnika Poznańska, Instytut Konstrukcji Budowlanych, ul. Piotrowo 5, 60-965 Poznań; tel. +48616652471; przemyslaw.wielentejczyk@put.poznan.pl

2 Roman Lewandowski, Politechnika Poznańska, Instytut Konstrukcji Budowlanych, ul. Piotrowo 5, 60-965 Poznań; tel. +48616652472; roman.lewandowski@ put.poznan.pl
} 
sowych. Porównano otrzymane krzywe rezonansowe z wynikami dla układów sprężystych z tłumieniem proporcjonalnym do macierzy mas [1].

\section{Sformułowanie problemu}

Analizuje się geometrycznie nieliniowe ustalone drgania belek wykonanych z materiału Kelvina-Voigta. Rozpatruje się belki jednoprzęsłowe o długość przęsła $L$. Drgania wzbudzane są przez dowolne siły harmonicznie zmienne w czasie o częstości wymuszenia $\lambda$. Schemat układu przedstawiono na Rys. 1. Belka na końcach posiada niepodatne podpory w kierunku poziomym. Symbolem $A$ oznaczono pole przekroju poprzecznego belki, $m$ masę rozłożoną na jednostkę długości, a $J$ moment bezwładności przekroju poprzecznego. Przemieszczenia poziome i pionowe belki oznaczono odpowiednio przez $u(x, t)$ i $w(x, t)$.

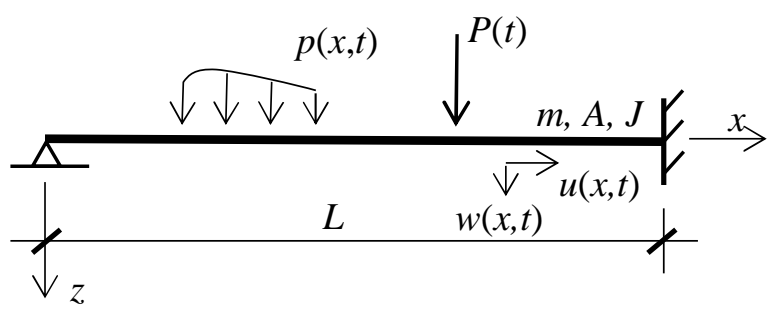

Rys. 1. Schemat belki jednoprzęsłowej

Fig. 1. Diagram of one-span beam

\subsection{Zależności geometryczne i związki konstytutywne}

Odkształcenia przekroju belki można zapisać następująco:

$\varepsilon_{x}(x, z, t)=\varepsilon(x, t)-z \kappa(x, t), \kappa(x, t)=-w,{ }_{x x}(x, t)$

gdzie: $\varepsilon_{x}(x, z, t)$ - odkształcenie dowolnego punktu przekroju poprzecznego o współrzędnej $z, \varepsilon(x, t)$ - odkształcenie osi obojętnej belki, $\kappa(x, t)$ - krzywizna belki, $(\cdot)_{, x}=d(\cdot) / d x$.

Zgodnie z teorią von Karmana można geometryczną nieliniowość problemu uwzględnić w następujący sposób:

$\varepsilon(x, t)=u,{ }_{x}(x, t)+\frac{1}{2} w,_{x}^{2}(x, t)$

Prawo konstytutywne dla materiału Kelvina-Voghta ma postać:

$$
\sigma_{x}(x, z, t)=E_{0} \varepsilon_{x}(x, z, t)+\tau E_{0} \dot{\varepsilon}_{x}(x, z, t)
$$


gdzie: $\tau$ - czas retardacji, $E_{0}$ - moduł sprężystości, $\sigma_{x}(x, z, t)$ - naprężenie normalne, $\dot{\varepsilon}_{x}=\mathrm{d} \varepsilon_{x} / \mathrm{d} t$.

Znając prawo konstytutywne opisane równaniem (3) można zapisać związki fizyczne za pomocą momentów zginających $M(x, t)$ i sił normalnych $N(x, t)$ oraz uogólnionych odkształceń w następujący sposób:

$$
\begin{aligned}
& N(x, t)=E_{0} A \varepsilon(x, t)+E_{0} A \tau \dot{\varepsilon}(x, t) \\
& M(x, t)=E_{0} J \kappa(x, t)+E_{0} J \tau \dot{\kappa}(x, t)
\end{aligned}
$$

Jeżeli pominie się wpływ osiowych sił bezwładności oraz założy niepodatność w kierunku osi belki podpór na końcach belki to równanie (4) można przekształcić do postaci

$$
N(t)=\frac{E_{0} A}{2 L} \int_{0}^{L} w,{ }_{x}^{2} \mathrm{~d} x+\tau \frac{E_{0} A}{L} \int_{0}^{L} w,_{x} \dot{w},{ }_{x} \mathrm{~d} x
$$

\subsection{Równania ruchu}

W pracy założono, że drgania układu wywoływane są przez siły harmonicznie zmienne, a w opisie tych sił uwzględnia się wyższe harmoniczne, tzn.:

$$
p(x, t)=p_{c 1}(x) \cos \lambda t+p_{s 1}(x) \sin \lambda t+p_{c 3}(x) \cos 3 \lambda t+p_{s 3}(x) \sin 3 \lambda t
$$

gdzie: $\lambda$-częstość wymuszenia, $p_{c 1}(x), p_{s 1}(x), p_{c 3}(x), p_{s 3}(x)$ - amplitudy siły wymuszającej.

W przypadku drgań ustalonych w ustalonej odpowiedzi układu również uwzględnia się wyższe harmoniczne. Zmiany w czasie przemieszczeń pionowych $w(x, t)$ oraz krzywizn $\kappa(x, t)$ przekroju opisuje się za pomocą funkcji:

$$
\begin{aligned}
& w(x, t)=w_{c 1}(x) \cos \lambda t+w_{s 1}(x) \sin \lambda t+w_{c 3}(x) \cos 3 \lambda t+w_{s 3}(x) \sin 3 \lambda t \\
& \kappa(x, t)=\kappa_{c 1}(x) \cos \lambda t+\kappa_{s 1}(x) \sin \lambda t+\kappa_{c 3}(x) \cos 3 \lambda t+\kappa_{s 3}(x) \sin 3 \lambda t
\end{aligned}
$$

Biorąc pod uwagę powyższe zależności i związki fizyczne (4) i (5) można siły normalne oraz momenty zginające zapisać w następujący sposób:

$$
M(x, t)=M_{c 1}(x) \cos \lambda t+M_{s 1}(x) \sin \lambda t+M_{c 3}(x) \cos 3 \lambda t+M_{s 3}(x) \sin 3 \lambda t
$$




$$
\begin{aligned}
N(t)= & N_{1} \cos ^{2} \lambda t+N_{2} \cos \lambda t \sin \lambda t+N_{3} \sin ^{2} \lambda t \\
& +N_{4} \cos ^{2} 3 \lambda t+N_{5} \cos 3 \lambda t \sin 3 \lambda t+N_{6} \sin ^{2} 3 \lambda t \\
& +N_{7} \cos \lambda t \cos 3 \lambda t+N_{8} \cos \lambda t \sin 3 \lambda t \\
& +N_{9} \sin \lambda t \cos 3 \lambda t+N_{10} \sin \lambda t \sin 3 \lambda t
\end{aligned}
$$

Amplitudy momentów zginających $M_{c 1}(x), M_{c 3}(x)$ i $M_{s 1}(x), M_{s 3}(x)$ można wyznaczyć stosując metodę bilansu harmonicznych podstawiając zależności (10) i (9) do równania (5), co prowadzi do następujących zależności:

$$
\begin{array}{ll}
M_{c 1}(x)=E_{0} J \kappa_{c 1}(x)+E_{0} J \tau \lambda \kappa_{s 1}(x) & M_{c 3}(x)=E_{0} J \kappa_{c 3}(x)+3 E_{0} J \tau \lambda \kappa_{s 3}(x) \\
M_{s 1}(x)=-E_{0} J \tau \kappa_{c 1}(x)+E_{0} J \tau \lambda \kappa_{s 1}(x) & M_{s 3}(x)=-3 E_{0} J \tau \lambda \kappa_{c 3}(x)+E_{0} J \kappa_{s 3}(x)
\end{array}
$$

W podobny sposób stałe występujące w (11) można wyznaczyć podstawiając równania (11) i (8) do równania fizycznego (6). Po żmudnych przekształceniach otrzymuje się:

$$
\begin{aligned}
& N_{1}=\frac{E_{0} A}{2 L} \int_{0}^{L} w_{c 1, x}^{2}(x) \mathrm{d} x+\tau \lambda \frac{E_{0} A}{L} \int_{0}^{L} w_{c 1, x}(x) w_{s 1, x}(x) \mathrm{d} x \\
& N_{2}=-\tau \lambda \frac{E_{0} A}{L} \int_{0}^{L} w_{c 1, x}^{2}(x) \mathrm{d} x+\frac{E_{0} A}{L} \int_{0}^{L} w_{c 1, x}(x) w_{s 1, x}(x) \mathrm{d} x+\tau \lambda \frac{E_{0} A}{L} \int_{0}^{L} w_{s 1, x}^{2}(x) \mathrm{d} x \\
& N_{3}=-\tau \lambda \frac{E_{0} A}{L} \int_{0}^{L} w_{c 1, x}(x) w_{s 1, x}(x) \mathrm{d} x+\frac{E_{0} A}{2 L} \int_{0}^{L} w_{s 1, x}^{2}(x) \mathrm{d} x \\
& N_{4}=\frac{E_{0} A}{2 L} \int_{0}^{L} w_{c 3, x}^{2}(x) \mathrm{d} x+3 \tau \lambda \frac{E_{0} A}{L} \int_{0}^{L} w_{c 3, x}(x) w_{s 3, x}(x) \mathrm{d} x \\
& N_{5}=-3 \tau \lambda \frac{E_{0} A}{L} \int_{0}^{L} w_{c 3, x}^{2}(x) \mathrm{d} x+\frac{E_{0} A}{L} \int_{0}^{L} w_{c 3, x}(x) w_{s 3, x}(x) \mathrm{d} x+3 \tau \lambda \frac{E_{0} A}{L} \int_{0}^{L} w_{s 3, x}^{2}(x) \mathrm{d} x \\
& N_{6}=-3 \tau \lambda \frac{E_{0} A}{L} \int_{0}^{L} w_{c 3, x}(x) w_{s 3, x}(x) \mathrm{d} x+\frac{E_{0} A}{2 L} \int_{0}^{L} w_{s 3, x}^{2}(x) \mathrm{d} x
\end{aligned}
$$




$$
\begin{aligned}
N_{7}= & \frac{E_{0} A}{L} \int_{0}^{L} w_{c 1, x}(x) w_{c 3, x}(x) \mathrm{d} x+3 \tau \lambda \frac{E_{0} A}{L} \int_{0}^{L} w_{c 1, x}(x) w_{s 3, x}(x) \mathrm{d} x \\
& +\tau \lambda \frac{E_{0} A}{L} \int_{0}^{L} w_{s 1, x}(x) w_{c 3, x}(x) \mathrm{d} x \\
N_{8}= & -3 \tau \lambda \frac{E_{0} A}{L} \int_{0}^{L} w_{c 1, x}(x) w_{c 3, x}(x) \mathrm{d} x+\frac{E_{0} A}{L} \int_{0}^{L} w_{c 1, x}(x) w_{s 3, x}(x) \mathrm{d} x \\
& +\tau \lambda \frac{E_{0} A}{L} \int_{0}^{L} w_{s 1, x}(x) w_{s 3, x}(x) \mathrm{d} x \\
N_{9}= & -\tau \lambda \frac{E_{0} A}{L} \int_{0}^{L} w_{c 1, x}(x) w_{c 3, x}(x) \mathrm{d} x+\frac{E_{0} A}{L} \int_{0}^{L} w_{s 1, x}(x) w_{c 3, x}(x) \mathrm{d} x \\
& +3 \tau \lambda \frac{E_{0} A}{L} \int_{0}^{L} w_{s 1, x}(x) w_{s 3, x}(x) \mathrm{d} x \\
N_{10}= & -\tau \lambda \frac{E_{0} A}{L} \int_{0}^{L} w_{c 1, x}(x) w_{s 3, x}(x) \mathrm{d} x-3 \tau \lambda \frac{E_{0} A}{L} \int_{0}^{L} w_{s 1, x}(x) w_{c 3, x}(x) \mathrm{d} x \\
& +\frac{E_{0} A}{L} \int_{0}^{L} w_{s 1, x}(x) w_{s 3, x}(x) \mathrm{d} x
\end{aligned}
$$

\subsection{Zastosowanie metody elementów skończonych}

Dyskretyzację rozpatrywanego problemu dokonano za pomocą metody elementów skończonych. Równanie pracy wirtualnej ma w rozważanym przypadku postać:

$$
\int_{0}^{L} \delta w(x, t)(b(x, t)+p(x, t)) d x-\int_{0}^{L}(\delta \kappa(x, t) M(x, t)+\delta \varepsilon(x, t) N(t)) d x=0
$$

Równania amplitud wyprowadzono stosując metodę bilansu harmonicznych. W tym celu użyto uśrednione w względem czasu równanie pracy wirtualnej. Ma ono postać:

$\frac{2}{T} \int_{0}^{T} \int_{0}^{L} \delta w(x, t)(b(x, t)+p(x, t)) d x d t=\frac{2}{T} \int_{0}^{T} \int_{0}^{L}(\delta \kappa(x, t) M(x, t)+\delta \varepsilon(x, t) N(t)) d x d t(15)$

gdzie $T=2 \pi / \lambda$ jest okresem drgań. 


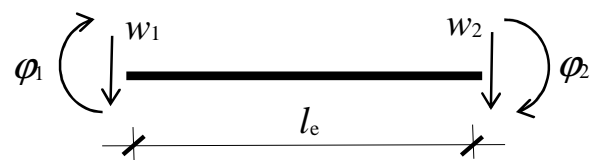

Rys. 2. Typowy element skończony i oznaczenie węzłowych przemieszczeń

Fig. 2. Typical finite element and signing of nodal displacements

W pracy zastosowano typowy element skończony o długości $l_{e}$ pokazany na Rys.2, dla którego aproksymację amplitud przemieszczeń i ich wirtualnych odpowiedników zapisuje się następująco:

$$
\begin{aligned}
& w_{c 1}(x)=\mathbf{H}(x) \mathbf{q}_{c 1 e}, w_{c 3}(x)=\mathbf{H}(x) \mathbf{q}_{c 3 e} \\
& w_{s 1}(x)=\mathbf{H}(x) \mathbf{q}_{s 1 e}, w_{s 3}(x)=\mathbf{H}(x) \mathbf{q}_{s 3 e} \\
& \delta w_{c 1}(x)=\mathbf{H}(x) \delta \mathbf{q}_{c 1 e}, \delta w_{c 3}(x)=\mathbf{H}(x) \delta \mathbf{q}_{c 3 e} \\
& \delta w_{s 1}(x)=\mathbf{H}(x) \delta \mathbf{q}_{s 1 e}, \delta w_{s 3}(x)=\mathbf{H}(x) \delta \mathbf{q}_{s 3 e}
\end{aligned}
$$

gdzie $\mathbf{H}(x)$ - wektor funkcji kształtu, którego składowymi są wielomiany Hermite'a trzeciego rzędu, $\mathbf{q}_{c 1 e}, \mathbf{q}_{c 3 e}, \mathbf{q}_{s 1 e}, \mathbf{q}_{s 3 e}$ - wektory węzłowych amplitud przemieszczeń.

Po żmudnych przekształceniach równania (15) i uwzględnieniu wzorów (12) i (16) można uzyskać nieliniowe równania amplitud w postaci:

$$
\begin{aligned}
\mathbf{r}_{c 1}= & \left(\mathbf{K}-\lambda^{2} \mathbf{M}\right) \mathbf{q}_{c 1}+\tau \lambda \mathbf{K} \mathbf{q}_{s 1}+\left(\frac{3}{4} N_{1}+\frac{1}{4} N_{3}+\frac{1}{2} N_{4}+\frac{1}{2} N_{6}+\frac{1}{4} N_{7}+\frac{1}{4} N_{10}\right) \mathbf{B} \mathbf{q}_{c 1} \\
& +\left(\frac{1}{4} N_{2}+\frac{1}{4} N_{8}-\frac{1}{4} N_{9}\right) \mathbf{B} \mathbf{q}_{s 1} \\
& +\left(\frac{1}{4} N_{1}-\frac{1}{4} N_{3}+\frac{1}{2} N_{7}\right) \mathbf{B} \mathbf{q}_{c 3}+\left(\frac{1}{4} N_{2}+\frac{1}{2} N_{8}\right) \mathbf{B} \mathbf{q}_{s 3}-\mathbf{P}_{c 1}=\mathbf{0} \\
\mathbf{r}_{s 1}= & \left(\mathbf{K}-\lambda^{2} \mathbf{M}\right) \mathbf{q}_{s 1}-\tau \lambda \mathbf{K} \mathbf{q}_{c 1}+\left(\frac{1}{4} N_{2}+\frac{1}{4} N_{8}-\frac{1}{4} N_{9}\right) \mathbf{B} \mathbf{q}_{c 1} \\
& +\left(\frac{1}{4} N_{1}+\frac{3}{4} N_{3}+\frac{1}{2} N_{4}+\frac{1}{2} N_{6}-\frac{1}{4} N_{7}-\frac{1}{4} N_{10}\right) \mathbf{B} \mathbf{q}_{s 1} \\
& +\left(-\frac{1}{4} N_{2}+\frac{1}{2} N_{9}\right) \mathbf{B} \mathbf{q}_{c 3}+\left(\frac{1}{4} N_{1}-\frac{1}{4} N_{3}+\frac{1}{2} N_{10}\right) \mathbf{B} \mathbf{q}_{s 3}-\mathbf{P}_{s 1}=\mathbf{0} \\
\mathbf{r}_{c 3}= & \left(\mathbf{K}-9 \lambda^{2} \mathbf{M}\right) \mathbf{q}_{c 3}+3 \tau \lambda \mathbf{K} \mathbf{q}_{s 3}+\left(\frac{1}{4} N_{1}-\frac{1}{4} N_{3}+\frac{1}{2} N_{7}\right) \mathbf{B} \mathbf{q}_{c 1} \\
& +\left(-\frac{1}{4} N_{2}+\frac{1}{2} N_{9}\right) \mathbf{B} \mathbf{q}_{s 1}+\left(\frac{1}{2} N_{1}+\frac{1}{2} N_{3}+\frac{3}{4} N_{4}+\frac{1}{4} N_{6}\right) \mathbf{B} \mathbf{q}_{c 3} \\
& +\left(\frac{1}{4} N_{5}\right) \mathbf{B} \mathbf{q}_{s 3}-\mathbf{P}_{c 3}=\mathbf{0}
\end{aligned}
$$




$$
\begin{aligned}
\mathbf{r}_{s 3} & =\left(\mathbf{K}-9 \lambda^{2} \mathbf{M}\right) \mathbf{q}_{s 3}-3 \tau \lambda \mathbf{K} \mathbf{q}_{c 3}+\left(\frac{1}{4} N_{2}+\frac{1}{2} N_{8}\right) \mathbf{B} \mathbf{q}_{c 1} \\
& +\left(\frac{1}{4} N_{1}-\frac{1}{4} N_{3}+\frac{1}{2} N_{10}\right) \mathbf{B} \mathbf{q}_{s 1}+\left(\frac{1}{4} N_{5}\right) \mathbf{B} \mathbf{q}_{c 3} \\
& +\left(\frac{1}{2} N_{1}+\frac{1}{2} N_{3}+\frac{1}{4} N_{4}+\frac{3}{4} N_{6}\right) \mathbf{B} \mathbf{q}_{s 3}-\mathbf{P}_{s 3}=\mathbf{0}
\end{aligned}
$$

gdzie $\mathbf{r}_{c 1}, \mathbf{r}_{c 3}$ i $\mathbf{r}_{s 1}, \mathbf{r}_{s 3}$ są wektorami reszt.

Globalne wektory obciążeń $\mathbf{P}_{c 1}, \mathbf{P}_{s 1}, \mathbf{P}_{c 3}, \mathbf{P}_{s 3}$ oraz globalne macierze mas $\mathbf{M}$, sztywności $\mathbf{K}$ i geometryczne $\mathbf{B}$ są agregowane w znany sposób przy użyciu macierzy definiowanych na poziomie elementu skończonego [2].

Macierzowo stałe $N_{1} . . N_{10}$ można przedstawić w następujący sposób:

$$
\begin{aligned}
& N_{1}=\frac{E_{0} A}{2 L} \mathbf{q}_{c 1}^{T} \mathbf{B} \mathbf{q}_{c 1}+\tau \lambda \frac{E_{0} A}{L} \mathbf{q}_{c 1}^{T} \mathbf{B} \mathbf{q}_{s 1} \quad N_{3}=-\tau \lambda \frac{E_{0} A}{L} \mathbf{q}_{c 1}^{T} \mathbf{B} \mathbf{q}_{s 1}+\frac{E_{0} A}{2 L} \mathbf{q}_{s 1}^{T} \mathbf{B} \mathbf{q}_{s 1} \\
& N_{2}=-\tau \lambda \frac{E_{0} A}{L} \mathbf{q}_{c 1}^{T} \mathbf{B} \mathbf{q}_{c 1}+\frac{E_{0} A}{L} \mathbf{q}_{c 1}^{T} \mathbf{B} \mathbf{q}_{s 1}+\tau \lambda \frac{E_{0} A}{L} \mathbf{q}_{s 1}^{T} \mathbf{B} \mathbf{q}_{s 1} \\
& N_{4}=\frac{E_{0} A}{2 L} \mathbf{q}_{c 3}^{T} \mathbf{B} \mathbf{q}_{c 3}+3 \tau \lambda \frac{E_{0} A}{L} \mathbf{q}_{c 3}^{T} \mathbf{B} \mathbf{q}_{s 3} \quad N_{6}=-3 \tau \lambda \frac{E_{0} A}{L} \mathbf{q}_{c 3}^{T} \mathbf{B} \mathbf{q}_{s 3}+\frac{E_{0} A}{2 L} \mathbf{q}_{s 3}^{T} \mathbf{B} \mathbf{q}_{s 3} \\
& N_{5}=-3 \tau \lambda \frac{E_{0} A}{L} \mathbf{q}_{c 3}^{T} \mathbf{B} \mathbf{q}_{c 3}+\frac{E_{0} A}{L} \mathbf{q}_{c 3}^{T} \mathbf{B} \mathbf{q}_{s 3}+3 \tau \lambda \frac{E_{0} A}{L} \mathbf{q}_{s 3}^{T} \mathbf{B} \mathbf{q}_{s 3} \\
& N_{7}=\frac{E_{0} A}{L} \mathbf{q}_{c 1}^{T} \mathbf{B} \mathbf{q}_{c 3}+3 \tau \lambda \frac{E_{0} A}{L} \mathbf{q}_{c 1}^{T} 1 \mathbf{B} \mathbf{q}_{s 3}+\tau \lambda \frac{E_{0} A}{L} \mathbf{q}_{s 1}^{T} \mathbf{B} \mathbf{q}_{c 3} \\
& N_{8}=-3 \tau \lambda \frac{E_{0} A}{L} \mathbf{q}_{c 1}^{T} \mathbf{B} \mathbf{q}_{c 3}+\frac{E_{0} A}{L} \mathbf{q}_{c 1}^{T} \mathbf{B} \mathbf{q}_{s 3}+\tau \lambda \frac{E_{0} A}{L} \mathbf{q}_{s 1}^{T} \mathbf{B} \mathbf{q}_{s 3} \\
& N_{9}=-\tau \lambda \frac{E_{0} A}{L} \mathbf{q}_{c 1}^{T} \mathbf{B} \mathbf{q}_{c 3}+\frac{E_{0} A}{L} \mathbf{q}_{s 1}^{T} \mathbf{B} \mathbf{q}_{c 3}+3 \tau \lambda \frac{E_{0} A}{L} \mathbf{q}_{s 1}^{T} \mathbf{B} \mathbf{q}_{s 3} \\
& N_{10}=-\tau \lambda \frac{E_{0} A}{L} \mathbf{q}_{c 1}^{T} \mathbf{B} \mathbf{q}_{s 3}-3 \tau \lambda \frac{E_{0} A}{L} \mathbf{q}_{s 1}^{T} \mathbf{B} \mathbf{q}_{c 3}+\frac{E_{0} A}{L} \mathbf{q}_{s 1}^{T} \mathbf{B} \mathbf{q}_{s 3}
\end{aligned}
$$

Rozwiązanie nieliniowego układu równań amplitud dla danej częstości wymuszenia $\lambda$ wymaga zastosowania metody kontynuacji opisanej np. w [2].

\section{Analiza dynamiczna belek}

W celu zbadania dynamicznych właściwości układów z materiału lepkosprężystego z uwzględnieniem wyższych harmonicznych przyjęto do analizy belkę wolnopodpartą pokazaną na Rys.3, która jako sprężysta była analizowana w pracy [1]. Drgania wzbudzane są przez siły harmonicznie zmienne w czasie 
zlokalizowane $\mathrm{w}$ odległości $L / 4$ od podpór o amplitudzie $P_{1}=13.63 E_{0} \mathrm{Jr} / \mathrm{L}^{3}$ i $P_{2}=-9.62 E_{0} J r / L^{3}$, gdzie $r$ oznacza promień bezwładności przekroju poprzecznego belki. Obliczenia wykonano dla równomiernego podziału belki na 8 elementów skończonych. Rezultaty prezentowane są w formie krzywych odpowiedzi jako funkcji względnej wypadkowej amplitudy przemieszczeń pionowych $w_{s} / h$ w punkcie przyłożenia siły wzbudzającej $P_{2}$ do bezwymiarowej częstości $\lambda / \omega_{1}$, gdzie $\omega_{1}$ jest podstawową częstością własną analizowanego układu. Wypadkowa amplituda $w_{s}$ jest pierwiastkiem sumy kwadratów składowych amplitud $w_{l c}$ i $w_{l s}$ w badanym punkcie, $h$ oznacza wysokość przekroju poprzecznego belki. Porównano krzywe odpowiedzi dla belek wykonanych z materiału sprężystego z tłumieniem proporcjonalnym do macierzy mas i współczynnika thumienia $c=0.01 \omega_{1}$ oraz modelu Kelvina-Voigta dla czasu retardacji $\tau=1 / 1500$ s. Charakterystyki materiału dla modelu sprężystego jak i lepkosprężystego dobrano tak aby wielkości maksymalnych amplitud przemieszczeń (rezonans) w badanym punkcie były podobne.

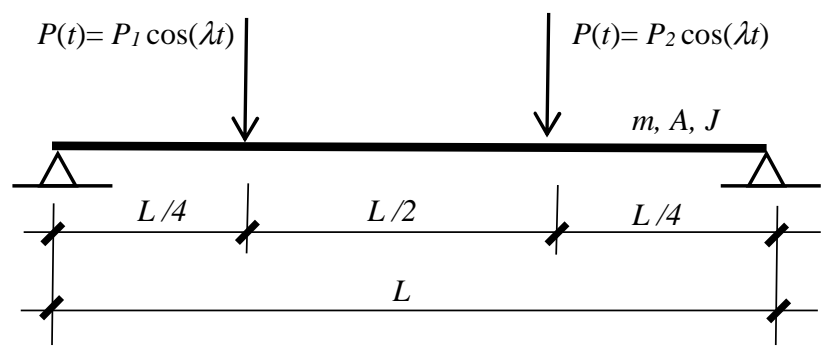

Rys. 3. Schemat analizowanej belki z niepodatnymi podporami na końcach

Fig. 3. Diagram of analyzed beam with immovable ends

Obliczenia wykonano dla następujących danych: rozpiętość przęsła belki $L=4.0 \mathrm{~m}$,wymiary przekroju poprzecznego $b \times h=0.4 \times 0.4 \mathrm{~m}$, moduł sprężystości materiału lepkosprężystego $E_{0}=7.0 \mathrm{MPa}$, zgodnie z pracą [3], jednostkowa masa belki $m=160 \mathrm{~kg} / \mathrm{m}$.

Na Rys.4 przedstawiono krzywe odpowiedzi dla obu badanych modeli materiału, sprężystego i lepkosprężystego Kelvina-Voigta. Można wyraźnie zaobserwować, że wyraźny rezonans wewnętrzny występujące tylko dla modelu sprężystego, natomiast dla modelu Kelvina-Voigta jest minimalne. Na Rys.5 powiększono strefę rezonansu wewnętrznego z Rys.4, która w przypadku dalszego obniżania czasu retardacji staje się coraz bardziej zauważalna. 


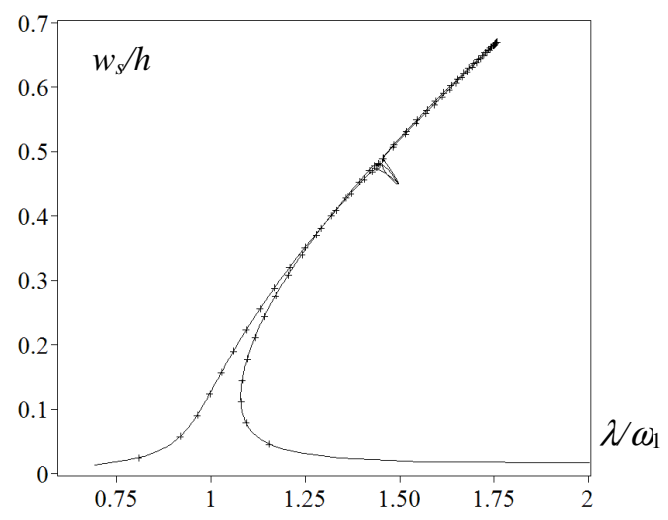

Rys. 4. Porównanie krzywych odpowiedzi dla belki swobodnie podpartej. Model sprężysty (- - ), model Kelvina-Voigta $(++++)$

Fig. 4. Comparison of response curves for simply supported beam. The elastic model (—_ Voigt model $(++++)$

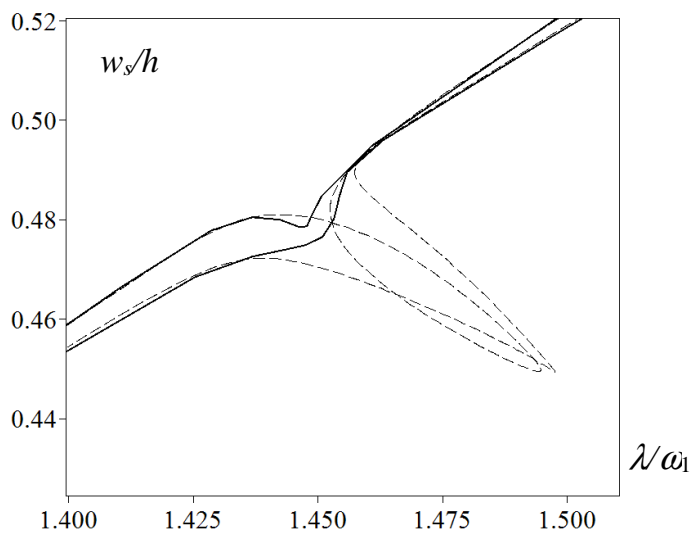

Rys. 5. Strefa rezonansu wewnętrznego. Model sprężysty (- - - ), model Kelvina-Voigta (- - )

Fig. 5. The internal resonance zone. The elastic model $(----)$, the Kelvin-Voigt model - -

\section{Wnioski}

W pracy zbadano wpływ wyższych harmonicznych na dynamiczne zachowanie się nieliniowych układów belkowych zbudowanych z materiału lepkosprężystego Kelvina-Voigta. Porównano wyniki z układem sprężystym z tłumieniem proporcjonalnym do macierzy mas. Wyraźnie wskazano, że w przypadku 
modelu materiału Kelvina-Voigta, pomimo bardzo niskiego czasu retardacji (małe tłumienie) nie dochodzi do zjawiska rezonansu wewnętrznego, co wskazuje, że wyraźnie wpływ na to zjawisko ma przyjęty model materiału oraz wpływ czasu retardacji na elementy nieliniowe równania amplitud, które nie występują w modelu sprężystym.

Podziękowania.

Badania byty wykonane w ramach działalności statutowej nr 01/11/DSPB/800.

\section{Literatura}

[1] Lewandowski R.: Okresowe drgania konstrukcji geometrycznie nieliniowych, Wydawnictwo Politechniki Poznańskiej, Rozprawy nr 291, Poznań 1993.

[2] Wielentejczyk P., Lewandowski R.: Geometrically nonlinear, steady state vibration of viscoelastic beams, International Journal of Non-Linear Mechanics, vol. 89, 2017, pp. 177-186.

[3] Galucio A.C., Deü J.-F., Ohayon R.: Finite element formulation of viscoelastic sandwich beams using fractional derivative operators, Computational Mechanics, vol. 33, 2004, pp. 282-291.

\section{INFLUENCE OF HIGHER HARMONICS ON NON-LINEAR VIBRATIONS OF BEAMS MADE OF KELVIN-VOIGT MATERIAL}

\section{S u m m a r y}

The problem of geometrically non-linear steady state vibrations of beams excited by harmonic forces is considered in this paper. The beams are made of a viscoelastic material defined by the classic Kelvin-Voigt rheological model. The von Karman theory and the Euler-Bernoulli theory are applied to describe the effects of geometric nonlinearities of beam deformations. The influence of axial inertial forces are neglected. The immovable ends of beam system are assumed. The physical relationships for internal forces and general deformations are defined. The equations of motion and amplitudes are derived using the virtual work method, the harmonic balance method and the finite element methodology. Non-linear amplitude equations are solved applying the continuation method. In the steady-state solution of equations of motion, the higher harmonics are taken into account what enables one to examine an secondary resonances but significantly complicates the formulation and solution of the problem. Using resonance curves the dynamic behaviour of beam systems made of the viscoelasticity Kelvin-Voigt material are presented. The results are compared with these ones for elastic beams. The significance of higher harmonics for the description of steady-state non-linear vibrations are briefly discussed.

Keywords: Kelvin-Voigt model, geometric nonlinearities of beam, FEM, steady-state analysis, higher harmonic analysis

Przestano do redakcji: 14.02 .2017 r.

Przyjęto do druku: 28.04.2017 r. 